\title{
Dissociation Between Visual Attention and Visual Mental Imagery
}

\section{Citation}

Thompson, William L., Yaling Hsiao, Stephen Michael Kosslyn. Forthcoming. Dissociation between visual attention and visual mental imagery. European Journal of Cognitive Psychology.

\section{Published Version}

http://www.informaworld.com/smpp/title db=all content=t713734596

\section{Permanent link}

http://nrs.harvard.edu/urn-3:HUL.InstRepos:4512992

\section{Terms of Use}

This article was downloaded from Harvard University's DASH repository, and is made available under the terms and conditions applicable to Open Access Policy Articles, as set forth at http:// nrs.harvard.edu/urn-3:HUL.InstRepos:dash.current.terms-of-use\#OAP

\section{Share Your Story}

The Harvard community has made this article openly available.

Please share how this access benefits you. Submit a story.

Accessibility 


\section{Dissociation Between Visual Attention and}

\section{Visual Mental Imagery}

William L. Thompson ${ }^{1}$, Yaling Hsiao $^{2}$, Stephen M. Kosslyn ${ }^{1,3}$

${ }^{1}$ Department of Psychology, Harvard University, Cambridge, MA 02138, USA

${ }^{2}$ Department of Psychology, University of Wisconsin-Madison, Madison, WI 53706, USA

${ }^{3}$ Department of Neurology, Massachusetts General Hospital, Boston, MA 02114, USA

Running head: Attention and imagery dissociation

Correspondence to:

William L. Thompson

Harvard University, Department of Psychology

844 William James Hall, 33 Kirkland Street

Cambridge MA 02138

USA

Tel.: 1-617-495-3932

Fax: 1-617-496-3122

Email: wt@wjh.harvard.edu

Keywords: mental imagery, visual attention, dissociation, visual mental representations, image size 
Visual mental imagery (which involves generating and transforming visual mental representations, i.e., seeing with the mind's eye) and visual attention appear to be distinct processes. However, some researchers have claimed that imagery effects can be explained by appeal to attention (and thus, that imagery is nothing more than a form of attention). In this study, we used a size manipulation to demonstrate that imagery and attention are distinct processes. We reasoned that if participants are asked to perform each function (imagery and attention) using stimuli of two different sizes (large and small), and that stimulus size affects the two functions differently, then we could conclude that imagery and attention are distinct cognitive processes. Our analyses showed that participants performed the imagery task with greater facility at a large size, whereas attention was performed more easily using smaller stimuli. This finding demonstrates that imagery and attention are distinct cognitive processes. 


\section{Dissociation Between Visual Attention and}

\section{Visual Mental Imagery}

Visual mental imagery involves creating, interpreting, and transforming visual internal representations (while "seeing with the mind's eye") whereas attention involves selecting some information for more detailed processing (while discarding other information). Thus, at first glance, the two functions appear distinct. Nevertheless, some researchers have claimed that mental imagery effects do not reflect characteristics of a distinct form of internal representation, but rather are best understood as products of attention. Pylyshyn (e.g., 1989, 2002, 2003), for example, has proposed that results from many "imagery" experiments actually reflect the allocation of attention to different portions of the space that would be occupied by an image. Pylyshyn (2002, p. 158) states that "...the use of visual indexes and focal attention provides a satisfactory explanation for how spatial properties are inherited from the observed scene, without any need to posit spatial properties of images." For example, when visualizing a house on a blank wall, they may think "the front door would be where the speck on the wall is", with attention's being allocated to different regions of space as the image is constructed descriptively.

Similarly, Pani (2002) has echoed Pylyshyn's view that phenomena attributed to mental imagery (such as the visualization of letters within a 4x5-cell grid) are actually due to the allocation of attention to defined regions of space. This idea gains credence because Craver-Lemley and Reeves (1992) have shown that imagery and attention can 
interact in some circumstances: in particular, when attention is divided, the Perky effect (i.e., the interference with perceiving that is produced by a mental image occupying the same space as a foveal visual percept) is half of that observed when attention is focused. If the Perky effect is taken as evidence that imagery and perception rely partly on the same neural systems, then the fact that divided attention decreases the effect whereas focused attention increases it could suggest that imagery effects arise, at least in part, from focused visual attention. We should note, however, that this effect of attention on imagery/perception interference only occurs when the perceptual targets are in the periphery of the visual field.

There is also increasing evidence that both visual imagery (e.g., Kosslyn \& Thompson, 2003; Kosslyn, Thompson \& Ganis, 2006) and visual attention can lead to increased activity in early visual cortex (e.g., Silver, Ress, \& Heeger, 2005), and attention may enhance performance of tasks that rely on this neural structure (e.g., Hopfinger \& West, 2006). These results make it difficult to disentangle effects due to imagery versus attention (but, see Offen, Schluppeck \& Heeger [2009] for evidence that attention and visual short-term memory rely on different processes in early visual cortex). In addition, Grossberg (2000) has suggested that a combination of mismatched attentional and topdown expectancy effects can give rise to the experience of perceiving a stimulus in its absence in the case of schizophrenic hallucinations.

However, Ishai, Haxby, and Ungerleider (2002) conducted a neuroimaging study in which they explicitly examined the effects of attention on imagery processing. They asked participants to visualize famous faces, and in one condition, asked them to focus their attention on a particular feature of the face (such as nose or lips). They found that 
requiring attention during imagery increased activation in some frontal and parietal regions (only a subset of areas activated by imagery), suggesting that the two processes are different. On the other hand, this result could be interpreted as showing that focusing attention to particular features represents higher resolution imagery to the target area, and so the study may not be considered a direct comparison of imagery and attention processes.

If attention and imagery rely on distinct mental processes, then there should exist some variables that affect imagery and attention differently. The size at which people are asked to perform an attention or an imagery task may be one variable that affects each function differently. In particular, for attention, there is evidence that people have more difficulty detecting faint visual signals if their attention is distributed over a large area than a small one (Eriksen \& St. James, 1986); conversely, for imagery, they have more difficulty detecting parts of a visualized object if the image is small than if the image is large (e.g., Kosslyn, 1975). Along the same lines, they also have poorer memory for objects visualized at small sizes versus large sizes (Kosslyn \& Alper, 1977). In the present study, we compare directly the effects of size in imagery versus attention using matched paradigms with the same participants .

If imagery and attention rely on the same processes, then manipulating the size of objects to be attended to or to be visualized should have the same effect. If, on the other hand, varying the size of the objects on which processing takes place produces different results for imagery and attention, then the two functions cannot rely on identical sorts of processing -- and we are justified in concluding that imagery cannot be reduced to attention. 


\section{Method}

Participants

The participants were 38 volunteers (17 females and 21 males) recruited from the Harvard University Department of Psychology Study Pool website. One female and five males were excluded from the study because they failed to understand the instructions in at least one task. Data from the 32 participants (16 females and 16 males) who successfully performed all tasks were retained for further analysis. The majority of the participants were Harvard undergraduate students or local residents, with a mean age of

23.5 years (range: 18 to 35 years). All reported normal or corrected-to-normal vision. All participants were compensated by payment of $\$ 10$ or awarding of course credits. The study was approved by the Harvard University Faculty of Arts and Sciences Committee on the use of Human Subjects and participants were tested according to all applicable guidelines and regulations governing the use of human participants in research.

\section{Materials}

Test stimuli were programmed into the PsyScope display program (Cohen, MacWhinney, Flatt, \& Provost, 1993) and were presented on a 15-inch Macintosh computer. Two conditions were administered to each participant: attention and imagery. In the attention condition, participants were asked to focus on a square at the center of the screen. The square could be presented at either a large or small size (see details below). Once participants had focused their attention on the square, a small dot appeared (on half the trials) somewhere within the boundaries of the square and participants were asked to indicate, by pressing a key on the computer keyboard, when they detected the appearance 
of the dot. The large square measured $16.5 \times 16.5 \mathrm{~cm}$ and the small square $1.8 \mathrm{X} 1.8 \mathrm{~cm}$. The squares had white interiors surrounded by a black frame. The black dot that could appear in the square measured approximately $1 \mathrm{~mm} \times 1 \mathrm{~mm}$. For each square, eight positions were defined where the dot might appear, and all were $2 / 3$ of the distance from the center to the edge of the square and of equal distance to each other. Figure 1 illustrates all the possible positions of the dots. The 48 trials were divided into two blocks of 24 trials. Each block consisted of a series of 24 trials that were to be performed at either a large or small size (using the appropriately sized square to set the area of attention or the size of the object to be visualized).

The design of the imagery condition was identical to that of the attention condition, except that, after the dot detection portion of the task, participants were asked to make an additional judgment about whether a common object or animal possessed a particular feature. Thus, two sound files were presented on each imagery trial: the first was the name of the object (to cue participants to visualize it) and the second the characteristic (on which participants judged the object they had visualized). To ensure that the time devoted to visualizing the objects would be similar across the set of objects, every sound file was recorded to last approximately one second. The length of each object name never exceeded six syllables.

For the imagery condition, we selected 48 common objects or animals from a larger set of approximately 100 objects. The investigators produced a list of common objects and, for each object, a property that the authors used imagery to verify (for example, "elephant" - "hanging ears"). These objects and their properties were pilot tested with a group of 26 college-age participants and we retained only those 
combinations where at least two thirds of the participants provided the correct response, indicating that they knew what the object was, could visualize it, and were able to visualize the property or characteristic in question. The questions were selected so that half were true (i.e, the property was in fact a characteristic of the object) and half were false (the property was not a characteristic of the object). The objects and accompanying properties were then divided into two groups of equal difficulty to be counterbalanced across size conditions of the study. The test items included animals, symbols, plants, signs, and cartoon characters. The properties included body parts, color, direction (e.g. direction of arrows of the recycling symbol), shape, position, and quantity (e.g. number of rings of the Olympic symbol). See Table 1 for a list of the items included in the study.

\section{Procedure}

Participants were tested individually in a small room with the lights on, and were seated at approximately $60 \mathrm{~cm}$ from the computer screen. Each received both the attention and imagery conditions, with the order counterbalanced over participants. A test session lasted approximately $60 \mathrm{~min}$, about half of which were spent on the computeradministered tasks and the rest to a set of written questionnaires. Each condition was composed of two blocks of trials, one where participants attended to (in the attention condition) or visualized within (in the imagery condition) an area defined by a large square and one block where they attended to or visualized within an area defined by a small square. The order of the size blocks was counterbalanced, so that half of the participants began with the block of "large" trials, while the other half began with the "small" trials. 
Thus, each participant completed four blocks in all: attention condition with large square, attention condition with small square, imagery condition with large square and imagery condition with small square. Each block started with detailed instructions and four practice trials to familiarize the participant with the task. To ensure that participants understood the instructions correctly, they were asked to paraphrase them to the investigator before beginning the practice trials. At that point, any necessary clarifications were made.

\section{Attention Condition}

Participants were asked to pay attention to an empty square (either large or small, depending on the block of trials) on the computer screen. They were asked to focus their attention on the square, and when they were ready (they were allowed as much time as they felt was needed to fully focus their attention) they pressed the spacebar. Once the spacebar was pressed, there was a delay, following which, on half the trials, a black dot would appear for $20 \mathrm{~ms}$. The exact timing of the dot's appearance was varied from trial to trial so that participants could not predict the specific time when the dot might appear; this required them to remain vigilant. Specifically, the delay (after the spacebar was

pressed) was either $1.5,2.0$, or $2.5 \mathrm{~s}$, and this timing was counterbalanaced across trials, where no more than two consecutive trials with dots had the same delay duration, and every duration occurred an equal number of times (approximately) in the first and the second half of a 24-trial block.

Participants were instructed to respond as quickly and accurately as possible by pressing the "yes" key ("yes" and "no" labels were affixed to the "b" and "n" keys 
respectively) on the keyboard as soon as they saw the dot. When no dot appeared, the empty square persisted for $4 \mathrm{~s}$ and another trial ensued. There was a $250 \mathrm{~ms}$ interval between trials. On trials where no dot appeared, participants were instructed simply not to respond. If the participants mistakenly thought that they had seen a dot (which in fact did not appear) and responded with the "yes" key within $4 \mathrm{~s}$, the trial would be terminated and followed by the next trial after a $250 \mathrm{~ms}$ interval. If the "yes" key was not pressed within $4 \mathrm{~s}$, the participant's response would be considered "no dot". The end of each trial was signaled by a beep. No more than 3 trials of the same type could occur in a row. On trials where a dot did appear, the location of the dot was counterbalanced within a block of trials. In addition, the same dot position never appeared in consecutive trials, and could only appear once or twice in each the first set of 12 and the second set of 12 trials of the block.

\section{Imagery Condition}

A judgment task based on the properties of the visualized objects was incorporated into the imagery condition in addition to the dot detection task. Participants were presented with an empty square at the beginning of each trial, either large or small depending on the block. After $1 \mathrm{~s}$, a word sound file named an object (e.g., "elephant"), which the participants were asked to visualize. They were instructed to create a vivid mental image that filled in the square without overflowing it, and to project that image onto the screen. The participants were to press the spacebar when a clear and vivid image had been completely generated. As in the attention condition, after 1.5, 2.0, or $2.5 \mathrm{~s}$, a dot

could appear briefly for $20 \mathrm{~ms}$ (the dot appeared on half of the trials). Participants were to press the "yes" key if they detected a dot or to wait four s without responding if they did 
not see a dot. The dot detection task ended once the "yes" key was pressed or after $4 \mathrm{~s}$ had passed without a response, with both situations being signaled by a beep. The participants were to maintain the vivid mental image of the cued object at all times while performing the dot detection task. After a $1 \mathrm{~s}$ delay, the participants heard a second word or phrase describing a characteristic that may or may not apply to the object that they were visualizing (e.g., "hanging ears"). Participants were instructed to decide whether the named characteristic pertained to the object by inspecting their mental image that had been projected on the square. The decision was indicated by pressing the "yes" or "no" key on the keyboard. Half of the trials required a "yes" response and the other half required a "no" response. The correct answers to the questions were never the same more for than three trials in a row. There were 6 questions with "yes" as the correct response and 6 with "no" as the correct response in each set of 12 trials within a block. No time limit was imposed on the task. A beep sound indicated that the response had been recorded and a new trial began $250 \mathrm{~ms}$ after each response.

The objects used in the imagery task were pilot-tested and divided into two groups that were equated for difficulty. The two sets of objects were counterbalanced over order and size of the image to avoid potential confounds.

\section{Questionnaires}

Following the computer-administered tasks, we asked the participants to complete a series of questionnaires on mental imagery, a personal health history to assess their vision and hearing, and the Edinburgh Handedness Inventory (Oldfield, 1971), which measures the preference in the use of hands for daily life activities. 


\section{Results}

We analyzed the time to detect the dots in both the attention and imagery conditions and the time to evaluate the properties in the imagery task. We first discarded response times (RTs) of incorrect responses and those that were greater than 2.5 standard deviations greater or smaller than the cell mean for that participant. Approximately $1 \%$ of the trials were excluded overall. The error rates for dot detection included trials where the participant missed the dot (misses) and where he or she incorrectly responded "yes" in the trials without a dot (false alarms). We also analyzed the time spent on focusing attention on the square for dot detection in the attention condition and that on generating the mental image of the named object in the square in the imagery condition. Finally, we calculated d' and beta for each participant for the dot detection task in both the attention and imagery conditions.

Size effects in attention versus imagery

We began by examining the data most relevant for our hypothesis. We conducted a 2 (imagery judgment vs. dot detection in the attention condition) x 2 (task performed at large vs. small size) ANOVA for both response times (RT) and error rates (ER).

RT analysis. Participants required less time in the dot detection task in the attention condition (398 ms) than they did to make judgments about objects they had visualized in the imagery condition $(1139 \mathrm{~ms}), \mathrm{F}(1,31)=75.95, M S E=230844, \mathrm{p}$ $<.0001$. No other main effects or interactions were significant.

ER analysis. Participants made fewer errors in detecting dots in the attention task when the size of the square was small $(0.9 \%)$ than when it was large $(6.3 \%)$, whereas in 
the imagery task (in which they judged characteristics of objects), they made fewer errors when the size of the square, onto which the image was projected, was large (15.4\%), than when the square was small (20.4\%), $\mathrm{F}(1,31)=37.3, M S E=0.002, \mathrm{p}<.0001$. Post-hoc contrasts revealed that the differences between each size pair (large and small) were significant in both the attention, $\mathrm{F}(1,31)=19.77, M S E=0.002, \mathrm{p}<.0001$, and the imagery, $\mathrm{F}(1,31)=17.56, M S E=0.002, \mathrm{p}<.0002$, tasks. See Figure 2 for an illustration of these results.

In addition to the interaction, we also found that participants made fewer errors in the attention condition overall (3.6\%) than in the imagery judgment condition overall $(17.9 \%), \mathrm{F}(1,31)=106.55, M S E=0.006, \mathrm{p}<.0001$.

\section{Dot detection in imagery versus attention}

Because smaller images are denser in their features and because visual images can interfere with like-modality perception, we conjectured that the dot detection effect (facilitation when attending to a small region of space) might be attenuated during the small-size imagery condition because the smaller, denser image might interfere more with the detection of the dot. Thus, we compared dot detection in the imagery and attention conditions, at the large and small size. We used a 2 x 2 ANOVA design.

$R T$ analysis. Participants required more time to detect the dot during the imagery condition $(532 \mathrm{~ms})$ than during the attention condition (398 ms), $\mathrm{F}(1,31)=92.3$, MSE $=6229, \mathrm{p}<.0001$. Participants required more time overall to detect the dot when the square was large ( $487 \mathrm{~ms})$ than when the square was small $(444 \mathrm{~ms}), \mathrm{F}(1,31)=27.6$, 
$M S E=27.62, \mathrm{p}<.0001$. The interaction between the two factors was not significant, $\mathrm{F}$ $(1,31)=0.48, M S E=2311$.

ER analysis. Participants made more errors in dot detection when the square was large $(6.5 \%)$ than when it was small $(1.7 \%), \mathrm{F}(1,31)=16.46, M S E=0.005, \mathrm{p}=.0003$. No other main effects or interactions were significant.

We found no effects of image generation time, nor of time to focus attention.

\section{Signal detection analysis}

Signal detection analyses allow us to consider both the participant's sensitivity to a given target (d') as well as any response biases toward "yes" or "no" responses (beta) when signaling that they have or have not detected the target.

Size effects in Attention and Imagery dot detection. We began with a 2 x 2 ANOVA comparing dot detection in the two conditions (attention and imagery) and at the two sizes (large and small square). We conducted separate analyses for $\mathrm{d}^{\prime}$ and beta as the independent variables. For d', the analysis revealed only that the participants had greater sensitivity to the dot when the square was small (mean $=3.3)$ rather than large $($ mean $=2.9), F(1,31)=17.78, M S E=0.225, \mathrm{p}=.0002$. No other main effect or interaction was significant. The same analysis for beta revealed a higher value (and thus, a greater bias toward "no" responses) in the imagery condition $($ mean $=0.18)$ than in the attention condition $($ mean $=0.12), \mathrm{F}(1,31)=5.92, M S E=0.02, \mathrm{p}<.03$. There was also a main effect for size, with participants showing higher beta values (a stronger tendency toward "no" responses) in the large $($ mean $=.22)$ rather than the small condition, (mean $=.08), \mathrm{F}(1,31), M S E=0.05, \mathrm{p}<.002$. These results may be explained by a greater 
number of misses in the imagery condition, compared to the attention condition, and in the large square condition compared to the small square condition. No other main effects or interactions were significant.

The analyses of the questionnaire data did not reveal any significant findings of theoretical importance, and thus we do not report those data here.

\section{Discussion}

In this study, we compared the effects of varying the size of the target on which we asked participants to perform either an attention or an imagery task. We reasoned that if the size manipulation affected the two types of functions differently, we could rule out the possibility that imagery and attention relied on the same type of processing. Our results confirmed that imagery and attention can be dissociated.

This result is not surprising, for many reasons. First, the claim that imagery is nothing more than focused attention fails to account for the many mental imagery results found when participants' eyes are closed (see, for example, Kosslyn, Thompson \& Ganis [2006] for a review). Although Pylyshyn's (1989, 1998, 2003) spatial indexing explanation might be considered plausible when eyes are open, it is unclear how such a system would work when no indexes were visible.

In addition, several studies have compared attention and imagery, and the results have suggested that they differ. For example, Slotnick, Thompson, \& Kosslyn (2005) showed that when participants are asked to visualize a flickering checkerboard wedge 
rotating, the retinotopic activation maps, as measured by functional magnetic resonance imaging (fMRI), more closely resemble retinotopic perceptual maps (resulting from actual viewing of the stimuli) than when participants are asked to pay attention to the region of space that would be occupied by the checkerboard.

We note that dot detection was less efficient in the imagery condition than in the attention condition. Though this result may, at first blush, appear to provide complementary evidence that the two functions are separate, there are other possible interpretations we must consider. This finding may be due to the increased effort or cognitive load required to perform two tasks at once (in the imagery task, participants are required both to detect the dot and to visualize and evaluate an image). Dot detection may require more time in such cases simply because performing both tasks is more effortful, and fewer resources can be devoted to each. Relatedly, participants may require more time to detect the dot during imagery because the cognitive load of performing both tasks is greater and this adds an additional level of executive processing.

In addition, this result is likely to represent a Perky effect, whereby visual imagery interferes with visual perception. The conditions found to be most favorable for the Perky effect to occur are present in this paradigm: Ishai and Sagi (1997) found that imagery/perception interference was greatest when images of common objects were formed from long-term memory (as was the case in our study) rather than short-term memory. Craver-Lemley and Arterberry (2001) showed that interference effects were strongest when the image overlapped the percept in a dot detection task. Indeed, our participants were instructed to fill the entire square, regardless of the size at which they were visualizing. Although, as mentioned above, attention has been found to influence 
the Perky effect under some conditions, there is consensus that the effect cannot be wholly explained by attention (Craver-Lemley \& Arterberry, 2001). The main effect of poorer dot detection during imagery is therefore inconclusive with respect to the relationship between attention and imagery. On the other hand, the interaction with size producing opposite effects in each function - is unambiguous and is strong evidence for the dissociation.

How, then, should we explain that attention and imagery may interact in some cases, as demonstrated by Craver-Lemley and Reeves (1992), or the visual indexing results shown by Pylyshyn (see, for example, Pylyshyn, 2003)? We propose that attention may be an important component in imagery generally -- one must, after all, pay attention to one's image and its component parts in order, for example, to inspect the image. Just as one would not replace the concept of "perception" with that of "attention" simply because attention is used in perception, one should not replace the concept of "imagery" with "attention" simply because attention plays a role in processing mental images. Moreover, attention may be particularly important in certain forms of imagery that rely on external visual stimuli and on spatial locations, such as when one pays attention to a tile floor and sees patterns of numbers formed by the tiles. However, when there are no external stimuli to guide one's image and the image features a complex shape that must be created from memory and either projected onto a blank screen or produced when the eyes are closed, then such images must be created on the basis of internal long-term memory representations - and this process may not be explained by appealing to attention allocation. It is difficult to explain, for example, how the complex features of a high-resolution image might arise from differential distribution of attention. How would 
attention represent complex features such as shape or texture? The results are clear: imagery and attention exist as distinct cognitive phenomena. 


\section{Acknowledgments}

This research was supported by Grant R01 MH060734 from the National Institute of Mental Health. Any opinions, findings, conclusions, or recommendations expressed in this material are those of the authors and do not necessarily reflect the views of the National Institute of Mental Health. Correspondence concerning this article should be addressed to W. L. Thompson, Department of Psychology, Harvard University, 844 William James Hall, 33 Kirkland Street, Cambridge, MA 02138 (e-mail: wt@wjh.harvard.edu). 


\section{References}

Cohen J.D., MacWhinney B., Flatt M., and Provost J. (1993). PsyScope: A new graphic interactive environment for designing psychology experiments. Behavioral Research Methods, Instruments, and Computers, 25, 257-271.

Craver-Lemley, C., \& Arterberry, M.E. (2001). Imagery-induced interference for a detection task. Spatial Vision, 14, 101-119.

Craver-Lemley, C., \& Reeves, A. (1992). How visual imagery interferes with vision. Psychological Review, 99, 633-649.

Eriksen, C. W., \& St. James, J. D. (1986). Visual attention within and around the field of focal attention: A zoom lens model. Perception and Psychophysics, 40, 225-240.

Grossberg, S. (2000). How hallucinations may arise from brain mechanisms of learning, attention, and volition. Journal of the International Neuropsychological Society, $6,583-592$.

Hopfinger, J. B., and West, V. M. (2006). Interactions between endogenous and exogenous attention on cortical visual processing. NeuroImage, 31, 774-789.

Ishai, A., Haxby, J. V., and Ungerleider, L. G. (2002). Visual Imagery of Famous Faces: Effects of Memory and Attention Revealed by fMRI. NeuroImage 17, 1729-1741 
Ishai, A., and Sagi, D. (1997). Visual imagery: Effects of short- and long-term memory. Journal of Cognitive Neuroscience 9:6, 734-742. Kosslyn, S. M. (1975). Information representation in visual images. Cognitive Psychology, 7, 341-370.

Kosslyn, S. M., and Alper, S. N. (1977). On the pictorial properties of visual images: effects of image size on memory for words. Canadian Journal of Psychology, 31, $32-40$.

Kosslyn, S. M., and Thompson, W. L. (2003). When is early visual cortex activated during visual mental imagery? Psychological Bulletin, 129, 723-746.

Kosslyn, S. M., Thompson, W. L., and Ganis, G. (2006). The Case for Mental Imagery. New York: Oxford University Press.

Offen, S., Schluppeck, D., and Heeger, D. J. (2009). The role of early visual cortex in visual short-term memory and attention. Vision Research, 49, 1352-1362.

Oldfield, R. C. (1971). The assessment and analysis of handedness: The Edinburgh inventory. Neuropsychologia, 9, 97-113.

Pani, J. R. (2002). Mental imagery is simultaneously symbolic and analog. Behavioral and Brain Sciences, 25, 205-206.

Posner, M.I. (ed) (2004). Cognitive Neuroscience of Attention. New York: Guilford

Pylyshyn, Z. W. (1989). The role of location indexes in spatial perception: A sketch of the FINST spatial-index model. Cognition 32, 65-97. 
Pylyshyn, Z. W. (1998). Thr role of visual indexes in spatial vision and imagery. In R. Wright (ed.), Visual Attention. New York: Oxford University Press, 215-231.

Pylyshyn, Z. W. (2002). Mental imagery: In search of a theory. Behavioral and Brain Sciences, 25, 157-238.

Pylyshyn, Z. W. (2003). Seeing and Visualizing: It's Not What You Think. Cambridge, MA: MIT Press.

Silver, M. A., Ress, D., and Heeger, D. J. (2005). Topographic maps of visual spatial attention in human parietal cortex. Journal of Neurophysiology, 94, 1358-1371.

Slotnick, S. D., Thompson, W. L., and Kosslyn, S. M. (2005). Visual mental imagery induces retinotopically organized activation of early visual areas. Cerebral Cortex, 15, 1570-1583. 
Table 1. List of imagery items and properties, divided in to "true" and "false" groups.

True

$\begin{array}{cc}\text { Elephant } & \text { hanging ears } \\ \text { American flag } & \text { blue stars } \\ \text { Cube } & 8 \text { corners } \\ \text { Star of David } & 6 \text { points } \\ \text { Cell phone keypad } & 0 \text { at bottom } \\ \text { Tic-tac-toe game } & 4 \text { lines } \\ \text { Kayak } & \text { two pointed ends } \\ \text { Stop sign } & 8 \text { sides } \\ \text { Bald eagle } & \text { hooked beak } \\ \text { Horse } & \text { pointy ears } \\ \text { Panda } & \text { black ears } \\ \text { Sea lion } & \text { flippers } \\ \text { Maple leaf } & \text { jagged edge } \\ \text { Penguin } & \text { white belly } \\ \text { Olympic symbol } & 5 \text { rings } \\ \text { Superman logo } & \text { red letter S } \\ \text { Mickey Mouse } & \text { gloves } \\ \text { Charlie Chaplin } & \text { mustache } \\ \text { Traffic light } & \text { red on top } \\ \text { Eiffel Tower } & 4 \text { legs } \\ \text { Face of 1-dollar bill } & \text { George Washington } \\ \text { Pepsi symbol } & \text { red on top } \\ \text { American flag } & \text { red stripe on top } \\ \text { Ronald McDonald } & \text { striped socks } \\ & \end{array}$


Table 1 (continued)

False

$\begin{array}{cc}\text { Pig } & \text { round ears } \\ \text { Donkey } & \text { hanging ears } \\ \text { Mercedes symbol } & 3 \text { curved lines } \\ \text { Parking meter } & \text { flat top } \\ \text { Washington Monument } & \text { dome } \\ \text { Statue of Liberty } & \text { torch in left hand } \\ \text { Elvis Presley } & \text { mustache } \\ \text { Chicken } & \text { webbed feet } \\ \text { Nike symbol } & \text { higher than wide } \\ \text { George Washington } & \text { beard } \\ \text { Ace of clubs } & 4 \text { leaves } \\ \text { Kangaroo } & \text { short tail } \\ \text { Honeycomb } & 5 \text { sides } \\ \text { Apple logo } & \text { bitten left side } \\ \text { Star fish } & 6 \text { points } \\ \text { Egyptian pyramid } & \text { triangular bottom } \\ \text { Fire Hydrant } & \text { square shape } \\ \text { Recycling symbol } & \text { counterclockwise arrows } \\ \text { American passport } & \text { wider than high } \\ \text { Starbucks symbol } & \text { triangular shape } \\ \text { Albert Einstein } & \text { glasses } \\ \text { Sphinx } & \text { standing } \\ \text { Cookie Monster } & \text { green fur } \\ \text { Winnie the Pooh } & \text { red nose }\end{array}$


Figure 1. A model of the type of square used in the attention and imagery conditions with dots at all 8 possible locations. The small square measured $1.8 \mathrm{~cm} \times 1.8 \mathrm{~cm}$ and the large square measured $16.5 \mathrm{~cm} \times 16.5 \mathrm{~cm}$. Both sizes of square were used in the imagery and attention conditions. The dots, which measured approximately $1 \mathrm{~mm} \times 1 \mathrm{~mm}$, remained at the same size regardless of whether the square was presented at a large or small size.

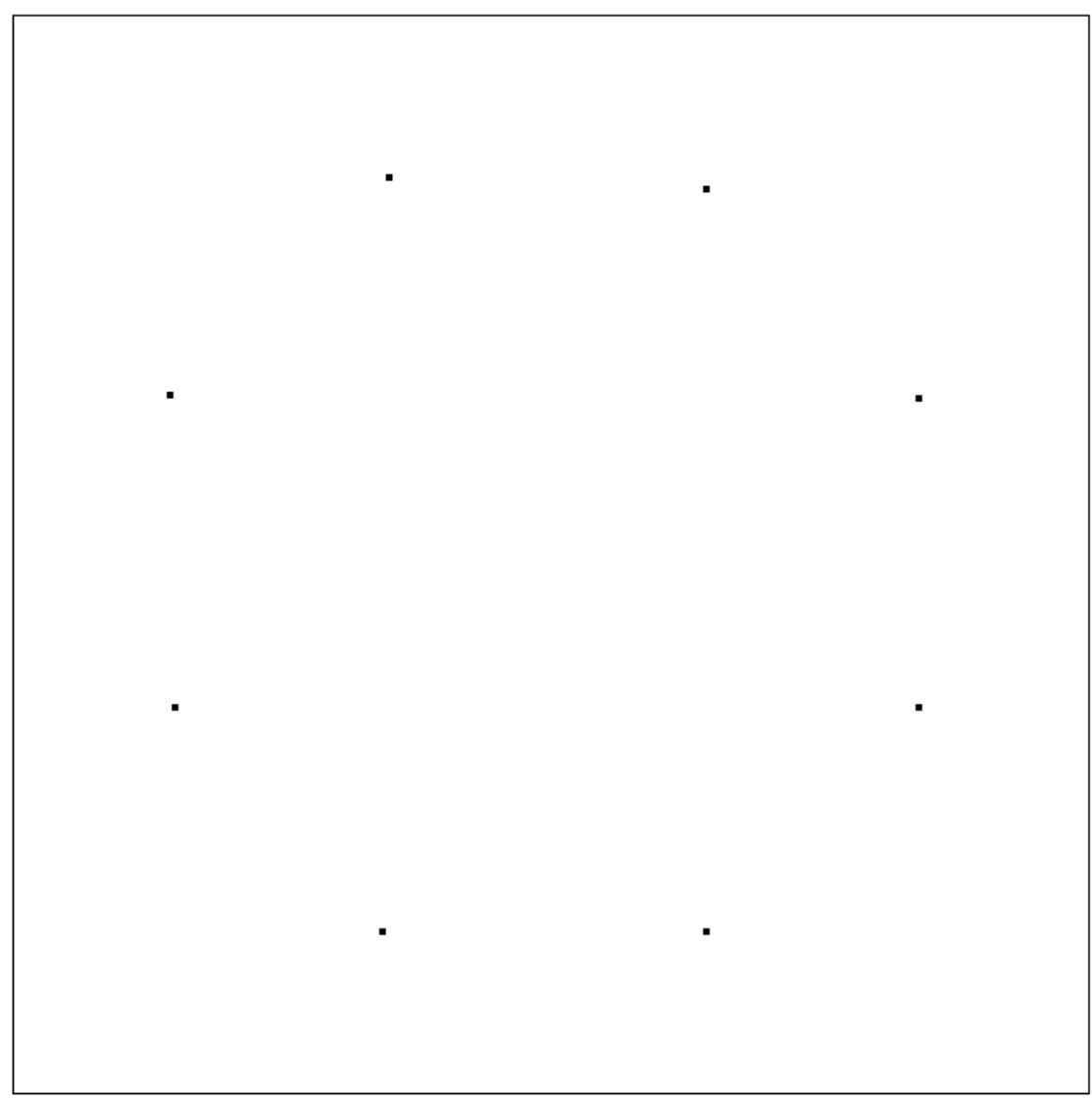


Figure 2. Errors in attention and imagery conditions performed at large and small size.

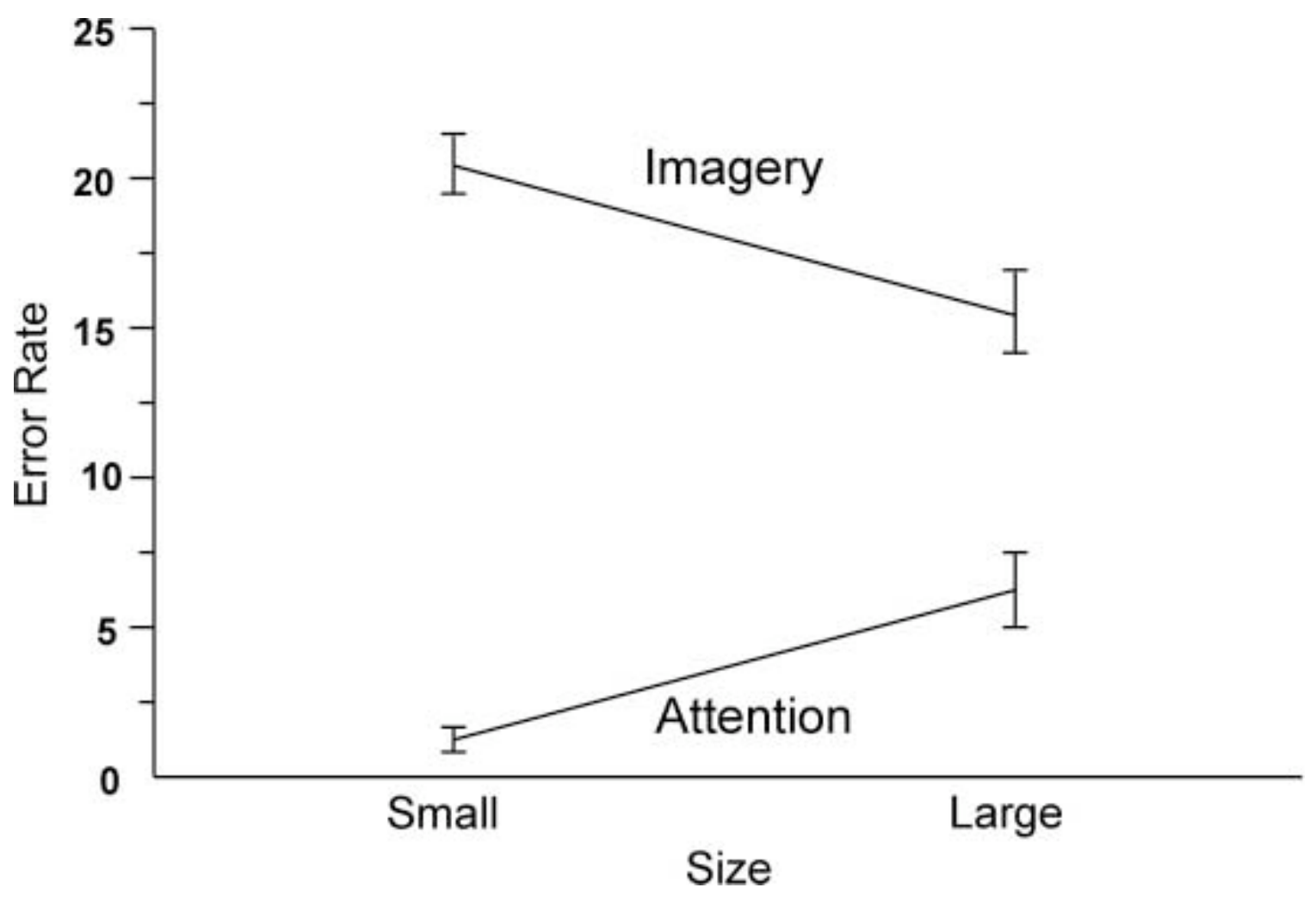

